\title{
Mobile consultant: evaluation of additional services
}

\author{
Konstantinos Banitsas ${ }^{1}$, Pantelis Georgiadis ${ }^{2}$, Sapal Tachakra ${ }^{3}$, Dionisis Cavouras ${ }^{2}$ \\ ${ }^{1}$ School of Engineering and Design, E\&CE, Brunel University, West London, England \\ ${ }^{2}$ Medical Image and Signal Processing Lab, Technological Educational Institution of Athens, Greece \\ ${ }^{3}$ A\&E department, North West London Hospitals, England
}

\begin{abstract}
As the need for mobility in the medical world increases, newer systems and applications came to light; many of them based on wireless and mobile networks.

PDA based systems were presented in the past, capable of videoconferencing and transmitting high quality images between a roaming consultant and a fixed point in the hospital. These systems not only had desirable characteristics but also incorporated additional services that were found of value: paging, Voice over IP calling, Internet, email, intranet, patient record update, etc

This paper presents an engineering and clinical evaluation of those additional services based on both objective and subjective criteria. It concludes that such complementary services can be desirable as they increase personnel mobility, utilize the hospital resources more efficiently while at the same time increase productivity and decrease the cost of hardware and communications.
\end{abstract}

Keywords - Telemedicine, PDA, VoIP, videoconferencing, wireless

\section{INTRODUCTION}

A $\mathrm{S}$ time passes doctors and medical consultants realize more and more the need for mobility: they do not have to be stationed servicing just a single area (usually within a hospital) but can freely roam within or outside the hospital premises while at the same time being reachable when needed.

As wireless and mobile networks are increasingly utilized, this need is fulfilled more and more each day: wireless networks are established inside the hospitals so medical devices can take advantage of those to allow communication between doctors and consultants.

Several such devices were introduced in the past [1]. MedLAN [2] was based on a wireless trolley capable of transferring real-time video and high quality still pictures to a medical consultant (usually residing inside the same hospital). This project rendered the old ISDN-based telemedical systems almost obsolete as they used too much space, had the need for expensive and slow ISDN connection and forced the relocation of the patient to specially equipped rooms.

Other systems utilized $3 \mathrm{G}$ communication links to offer accessibility anywhere [3], [4]. They were able to send biosignals, video and images onto a fixed consultation point, while maintaining their mobility.

Last year, a newer system was introduced, capable of performing all of the above while being based on a small and lightweight PDA system [5]. This PDA could perform videoconferencing, send and receive high-resolution still images while also supporting a variety of additional services like paging, VoIP, intranet, Internet and email access, patient record update, etc. As the most desirable characteristics of the system at the time of development seemed to be the real-time video and audio conferencing, the clinical evaluation was limited only in those two applications. However, as the system was used more and more, the value of the additional services emerged along with the need for their proper evaluation.

What was asked by the doctors and consultants, was a system that could deliver phone services using a wireless hospital infrastructure, would replace the conventional hospital pagers and will increase the number of computer services within a hospital that a doctor can access (e.g. patient records or the intranet / Internet). All that had to fit in a small PDA that would have sufficient computational power to carry out these operations while being easily manageable, simple to use, small to carry and cheap to run [Fig. 1].

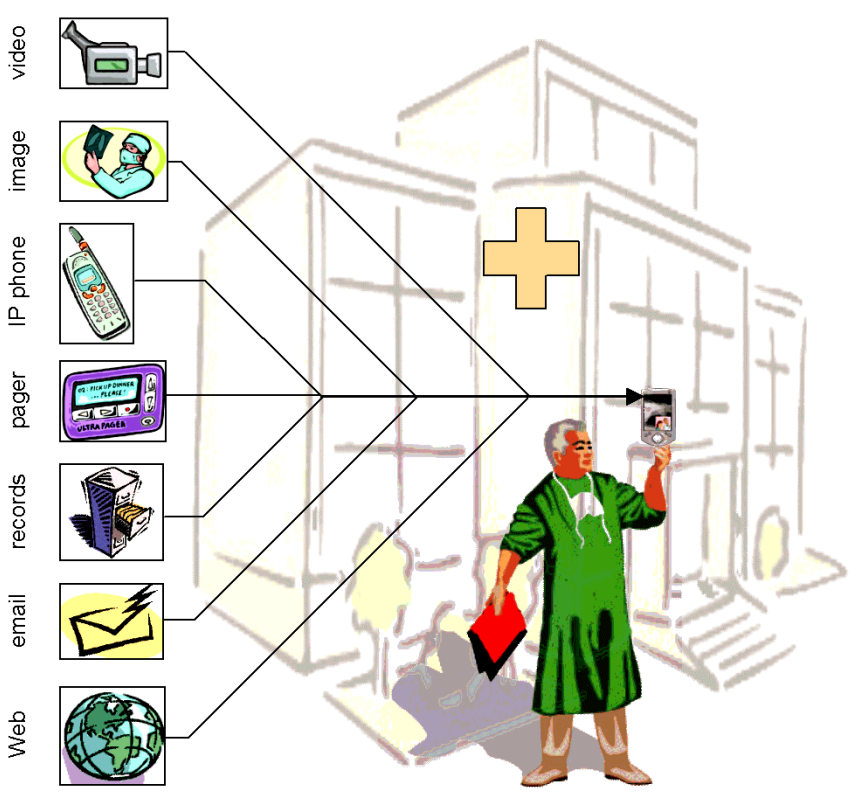

Figure 1. All in one. Using a PDA to implement a variety of services

\section{Methodology}

During the evaluation phase of the proposed system, several Access Points (APs) where placed in and around the 
Accidents and Emergency ward (A\&E) of Central Middlesex Hospital, London. As IEEE $802.11 \mathrm{~b}$ and $\mathrm{g}$ support for three independent channels, the APs were placed in such a way that the range of each did not interfere with the range of the other using the same frequency. In that sense, the network could extent infinitely in a cell-like structure. The area of majors and minors injuries was covered, along with the corridors around that ward and the A\&E offices. The objective was to provide for a wireless network that will run on top of the existing, being connected to the wired one but not consuming any significant amount of bandwidth. The doctor / consultant should be able to freely move around the area supported by the APs and handover from one cell to the other without loosing connection.

Next, there was the choice of the mobile hardware. The HTC series (both the Universal and Advantage models) proved to be ideal candidates. They supported CPUs running at speeds more than $520 \mathrm{MHz}, 2-4 \mathrm{~GB}$ memory and an excellent display with a resolution of $480 \times 640$ pixels. They both supported quad band mobile networks (allowed for video calling) alongside Bluetooth and IEEE $802.11 \mathrm{~b}$ WLAN. Their operating system was WM5 and WM6 respectively; a prerequisite in order to use Microsoft's SDK. All standard programs (Word, Excel, PowerPoint, pdf reader) were embedded in both of those PDAs.

Apart from the videoconferencing and image viewing / manipulating scenario that was already tested [5] the following scenarios were put to the test:

- Paging: replacing the conventional pagers that can only accept simple text, with a more advanced paging system supported by the above-mentioned PDA. In that scenario, the consultant can also get a variety of multimedia information while being paged, including high resolution images, full patient data, video of patient's vital signs or injuries, ECG, etc. The objective was to create an instant email-like pager that will have no limits on the amount or the type of data to be sent.

- Virtual IP-phone: It is obvious that there is a shift from conventional circuit switched phones into an all-IP technology. The reasons for that are numerous and include significant reductions of cost, additional functionality, instant number portability, etc. For this research project, a soft-phone was build to use the SIP protocol (Session Initiation protocol) over the existing wireless LAN. This way, the PDA could be assigned a user defined, landline number from any area of the world and by having a simple application run in the background could react as a wireless telephone for both performing incoming and outgoing calls, wherever there is an Internet connection. The whole procedure involved digitizing the sound with any of the wellknown audio compression codecs, passing the digitized audio through the intranet / internet lines onto a SIP server and from then on to any SIP or conventional circuit-switched phone (POTS). The user has a choice on the audio codec to be used: either the uncompressed G.711 needing a constant stream of $64 \mathrm{Kbps}$ or the compressed G.729 occupying only 8 Kbps. Overall, the system's behavior should be comparable to a landline phone call with some additional delay due to compression and IP routing delays.

In both the above-mentioned applications the software was build from zero: Microsoft Visual C++ 2005 was used to develop the applications under the Microsoft Windows Mobile 5 operating system. The software occupies only 4.5 MB of memory and while being in stand-by mode uses only $3 \%$ of CPU time.

- Accessing patient record and intranet: As the PDA is connected to the WLAN and from then on to the hospital network, it can enjoy the same privileges as a desktop computer including network access and access to shared devices, printing, storing, etc. It even has a proper QWERTY keyboard for easy data entry.

- Internet / email access: inside a hospital often the need for terminal access is high, especially in places like A\&E. Having a PDA to wirelessly access Internet resources or check emails was put to the test, both in terms of speed but mostly in terms of functionality and ease of use.

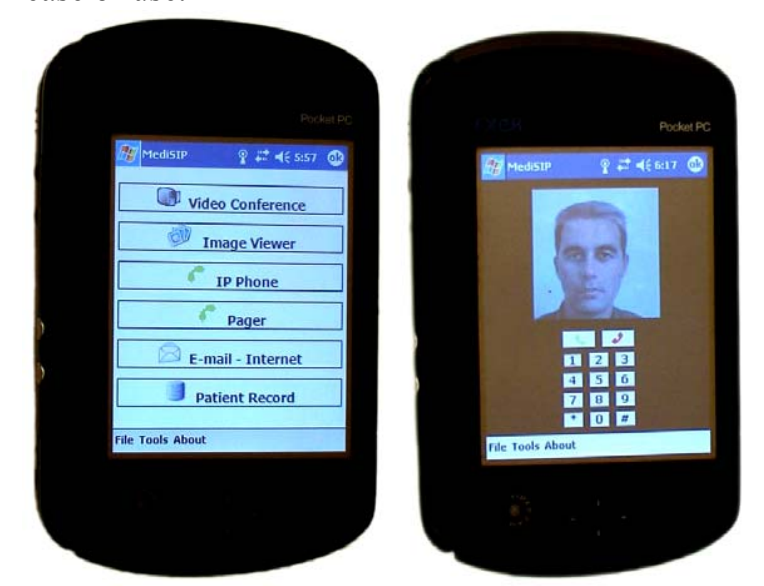

Figure 2. Main menu on the PDA and soft VoIP phone application

The methodology called for three consultants and doctors using the system as much as possible over a period of two weeks and evaluate its performance mostly using subjective criteria over a total of 50 cases. The criteria included clarity of image and sound, ease of use, time saved compared to conventional means (if any), etc. Two communication engineers used the system at the same time and evaluated its general QoS characteristics including stability, packet lost, loss of communication, end-to-end delay, jitter, bandwidth used, speed of accessing the information, etc.

\section{RESULTS}

The behavior of the PDA under videoconferencing and image sending was discussed in detail in a past paper [5] and 
will only be presented in brief: The PDA was capable of videoconferencing in a number of resolutions ranging between $176 \times 144$ and $352 \times 288$ pixels. Although the necessary bandwidth for the highest resolution was available without any major dropping of the frame rate, it was rarely used, as it will impose an additional load to the wireless network that might prevent other people accessing it. The frame rate varied between 5 and 15 frames per second, depending on the distance between the mobile user and its associated AP (802.11b connection speed falls back to 5.5, 2 and $1 \mathrm{Mbps}$ as the signal deteriorates). When the movement of the source camera increased, the image on the receiving end appeared relatively "squared" but this is to do with the way that the compression algorithm behaves [6].

High-resolution images of various sizes could be wirelessly sent to the mobile unit. Zooming and panning was easily performed using either the PDA's pen or one's finger. The average time for a $500 \mathrm{~KB}$ image to be sent over to the consultant varied between 2-5 seconds.

What is of more interest is the behavior of the additional services that the whole system supported with emphasis in the real time applications like the virtual IP-phone. Below, these services are listed along with their measurements in respect to the QoS.

Pager: the paging system was not exactly a real-time application but mostly a store-and-forward system. The sender used a piece of software that was developed by the research team to incorporate any kind of information he / she wished to be sent to the mobile PDA. Accordingly, the size of the file to be sent varied from a single $\mathrm{KB}$ to lots of $\mathrm{MB}$ with an average of about 500KB (mostly image and text). In order for the mobile PDA to receive this message it had to use a process that remained active in the background of the PDA's OS and would react every time a paging message had been sent. The overall end-to-end delay was negligible varying between $0.5-3 \mathrm{sec}$.

It is worth noticing that the design of the system allowed for storing of the message whenever the recipient was unavailable. This was based to a technique similar to those of SMS messaging: The network will initiate a search of the device in question and if it finds it will deliver the message and the recipient will issue an acknowledgement. If not (the user is not within AP range or is switched off), the network will retry in user-defined, regular intervals (every five minutes in this case) before quitting after a user-defined period of time (two days).

Virtual IP-phone: there can be many ways of exploiting a wireless VoIP phone; the most effective one would be to assign a geographical number into that which can be dialed from anywhere. Although many hospitals can have their own VoIP servers that support SIP protocol, CMH was an exception and for reasons of simplicity an external communication provider was chosen. so the PDA behaved as a cordless phone whenever it was within the range of the APs [Fig. 2].
Connection establishment delay was practically zero for internal calls and 2-5 seconds for external circuit switched calls. Failure probability over the period of test was $6 \%$. Most of the times it involved roaming from one AP to another. This handover introduced an inevitable delay of about 1-5 seconds due to the AP disassociation / reassociation procedure.

The bandwidth that was used was heavily depended on the codec: ITU-T's G.711 uses 64Kbps and uncompressed data. If the communication channel is relatively clear it outperforms all other codecs. However, if there is traffic in the channel, it is likely to cause additional delays. ITU-T's G.729 crates a user-defined buffer (60ms in this case) and compresses the stream to $8 \mathrm{Kbps}$ [7], [8]. This, along with some predictive coding, competes adequately with G.711 in terms of quality, while at the same time behaving better under most conditions.

Transit delay, or end-to-end delay was, again, codec dependant. Under typical circumstances compressed codecs can have less delay due to their reduced bandwidth demand: there are fewer bottlenecks in the network and thus fewer delays. In general, G.711 delay was about 100-600 ms while G.729 was about $250-500$ ms with a jitter of about $100 \mathrm{~ms}$ on an average hospital network with $5-20 \%$ utilization. One should keep in mind that the above figures include wireless network, wired network, routing and server delays.

Hospital network / patient record: the system behaves similarly to a desktop station accessing the hospital network. It can open and save files of all types and access patient record for viewing and altering. At this initial stage, the system could only access intranet-based patient records. However, through SDK it can be programmed to be compatible with most of the patient record systems used at hospitals today. Furthermore, it can even view and manipulate DICOM images although the screen resolution of 640 × 480 pixels was proven sometimes inadequate (DICOM specifications include 640 x 480 standard, lossless or lossy, for ultrasound but require considerably more resolution for $\mathrm{x}$-rays and $\mathrm{CT} / \mathrm{MRI}$ ) [9]. For these cases, panning and zooming was used.

Best compatibility is achieved if the hospital network is based on intranet / web page design. Speeds and delays on that scenario follow those of the hospital network and are capped only on the WLAN ceiling mentioned next.

Internet / email access: this was the most conventional scenario as the PDA supported that internally. Microsoft Windows Internet explorer and Microsoft Outlook were used without major problems. As WM5 is not fully compatible with Microsoft Windows, there was a small percentage of web pages that were not displayed properly mainly because of their size (PDA support a default resolution of $640 \times 480$ ). There were no problems reported on sending / receiving emails and attachments. The bandwidth available for browsing and accessing depended on the hospitals network and can practically reach 2-3 Mbps 
on an $11 \mathrm{Mbps}$ IEEE $802.11 \mathrm{~b}$ and about $15-25 \mathrm{Mbps}$ on an $802.11 \mathrm{~g}$ network, depending on the distance and the signal strength of the AP.

\section{DISCUSSION}

Overall, the suggested system behaved satisfactory while having a modest cost of about 700 Euros.

As far as the feedback from the clinicians is concerned and for the additional services of this project, their opinion was recorder for all cases that run during the testing phase using subjective criteria: image clarity, sound clarity, delay, ease of use, diagnostic value, diagnostic accuracy (even though this tool is not indented as a complete diagnostic tool). The results are summarized in Table I.

TABLE I

CLINICIAN'S FEEDBACK (\%)

\begin{tabular}{lccc}
\hline \hline \multicolumn{1}{c}{ Factor } & Good & Acceptable & $\begin{array}{c}\text { Not } \\
\text { acceptable }\end{array}$ \\
\hline Image clarity & 86 & 8 & 6 \\
Sound clarity & 95 & 5 & 0 \\
Communication delay & 92 & 8 & 0 \\
Diagnostic value & 70 & 20 & 10 \\
Diagnostic accuracy & 82 & 12 & 6 \\
Ease of use & 66 & 20 & 14
\end{tabular}

It is expected that it would need some time for any doctor or consultant to get used in such technology and find it relatively easy to use.

In engineering terms it is worth mentioning the following:

The range of the IEEE $802.11 \mathrm{~b}$ cells was greater that those of $802.11 \mathrm{~g}$ [10]. Respectively, more clients were able to use an $802.11 \mathrm{~b}$ cell thus creating more traffic per cell. As the traffic approached about $50 \%$ of its practical limit (2-3 and $15-25 \mathrm{Mbps}$ on $802.11 \mathrm{~b}$ and g respectively) there were obvious delays in the network, especially during real-time applications, which means that there is a limit on the devices that can be used at the same time. Depending on the applications that these devices run, this number can very between 4 and 15 PDAs communicating simultaneously. The problem can be addressed by placing more APs (preferably using $802.11 \mathrm{~g}$ architecture) while covering a smaller space.

Security is also of great concern as most of the times there is transfer of sensitive personal information. It was made clear from the start that doctors and consultants would not be able to use this product unless every possible effort was made to secure the over-the-air channel that data was transferred and comply with the privacy and confidentiality directives set by the local health system.

The overall security of the system relies both on the use of wireless encryption (WEP) and use of memorable information (username / passwords). Wired Equivalent Privacy (WEP) was repeatedly proven weak due to the implementation of the RC4 encryption algorithm [11]. Even security algorithms like WPA (Wi-Fi Protected Access) can be compromised by brute force attack. A viable solution would be to use WEP or WPA [12] as encryption, use MAC address filtering to control the wireless terminals accessing the networks and also use authentication servers like RADIUS, especially for the in-hospital communications. This way, the user will be prompted for a username / password at the start of the session (e.g. start of the day) which will then be compared to a list of nominal users.

\section{CONCLUSION}

In this paper we have presented a system that apart from being capable of real time videoconferencing and medical image transmission, can also perform a variety of value added services that can be proven beneficiary to the patient treating procedure. All the medical needs, as described in the introductory section, were either met or exceeded. Furthermore, the clinical testing of those services prove that it can speed up communication between treating doctors and consultants, provide new and desirable services while at the same time economize on hospital resources.

\section{REFERENCES}

[1] Bhargav Raman, et. al., "Radiology on handheld devices: images display, manipulation, and PACS integration issues", Radiographics, Vol. 24, No.1, pp. 299-310, Jan-Feb 2004

[2] Konstantinos A. Banitsas, et. al., "Applications of Medical Wireless LAN Systems (MedLAN)", International Journal for Medical Marketing, vol. 2, no. 2, pp. 136-142, Jan 2002

[3] Yuechun Chu, Aura Ganz, "A mobile teletrauma system for rural trauma care", $26^{\text {th }}$ EMBC, San Francisco, 2004, p. 3282-3285

[4] Konstantinos A. Banitsas, et. al., "Using 3G links to develop a teleconsultation system between a moving ambulance and an A\&E base station", International Journal of Telemedicine and Telecare, vol. 12, p. 23-25, Royal Society of Medicine press, 2006

[5] Konstantinos A. Banitsas, et. al., "Mobile Consultant: combining total mobility with constant access", IEEE EMBC 2006, p. 52485251, Sep 2006, N.Y., N.Y.

[6] Abdul Sadka, "Compressed Video Communications", John Wiley \& Sons, 2002

[7] A. Benyassine, E. Shlomot, H. Y. Su, D. Massaloux, C. Lamblin, and J. P. Petit, "ITU-T Recommendation G.729 Annex B: a silence compression scheme for use with G.729 optimized for V.70 digital simultaneous voice and data applications", Communications Magazine, IEEE, vol. 35, pp. 64-73, 1997.

[8] R. Salami, C. Laflamme, B. Bessette, and J. P. Adoul, "Description of ITU-T Recommendation G.729 Annex A: reduced complexity 8 kbit/s CS-ACELP codec", in 1997 IEEE International Conference on Acoustics, Speech, and Signal Processing (ICASSP-97), 1997, pp. 775-778 vol.2.

[9] National Electrical Manufacturers Association, "Digital Imaging and Communications in Medicine (DICOM) Part 5: Data Structures and Encoding", NEMA, PS 3.5-2007

[10] Banitsas A. Konstantinos, Sapal Tachakra, Robert S. H. Istepanian, "Operational Parameters of a Medical Wireless LAN: Security, Range and Interference issues", Presented at IEEE EMBS conference 2002, vol. 3, pp. 1889-1890, Oct 2002, Houston

[11] Scott Fluhrer, Itsik Mantin, and Adi Shamir, "Weaknesses in the Key Scheduling Algorithm of RC4", 8th Annual International Workshop on Selected Areas in Cryptography, pp. 1-24, Springer-Verlag, London, 2001

[12] Norio nakata, et. al., "Mobile wireless DICOM server system and PDA with high-resolution display", Radiographics, Vol.25 No.1, pp273-283, Jan-Feb 05 